\title{
Selection of Amino Acid Chirality via the Stark Effect in Stellar Environments ${ }^{+}$
}

\author{
Michael Famiano ${ }^{1, *}$, Toshitaka Kajino ${ }^{2,3}$ and Richard Boyd ${ }^{2}$ \\ Department of Physics, Western Michigan University, Kalamazoo, MI 49008, USA \\ 2 Division of Theoretical Astronomy, National Astronomical Observatory, National Astronomical \\ Observatory of Japan, Tokyo 113-0033, Japan; kajino@nao.ac.jp (T.K.); richard11boyde@comcast.net (R.B.) \\ 3 Department of Physics, University of Tokyo, Tokyo 113-8654, Japan \\ * Correspondence: michael.famiano@wmich.edu \\ † Presented at Symmetry 2017-The First International Conference on Symmetry, Barcelona, Spain, \\ 16-18 October 2017. \\ Published: 3 January 2018
}

An astro-biological model is presented in which atomic nuclei bound in amino acids interact via the weak interaction in stellar environments. Amino acids are preferentially oriented in high magnetic fields of certain stellar environments via the Stark effect. The coupling of atomic nuclei with non-zero magnetic moments to the molecular orbitals via the hyperfine interaction creates additional energy splittings. It has also been found that the magnetic shielding tensor can create an asymmetry in the energy states of the molecules which is dependent on chirality, and this is exploited in this model to create a chirality-dependent asymmetry in molecular states. An enantiomeric excess is subsequently created via the selective destruction and subsequent amplification of nuclei oriented in strong fields. Possible sites are proposed in which this model may exist.

Conflicts of Interest: The authors declare no conflict of interest.

(C) 2018 by the authors. Licensee MDPI, Basel, Switzerland. This article is an open access article distributed under the terms and conditions of the Creative Commons Attribution (CC BY) license (http://creativecommons.org/licenses/by/4.0/). 